\title{
The New Mittag-Leffler Function and Its Applications
}

\author{
U. Ayub, ${ }^{1}$ S. Mubeen, ${ }^{1}$ T. Abdeljawad ${ }^{2,3,4}$ G. Rahman ${ }^{2},^{5}$ \\ and Kottakkaran Sooppy Nisar $\mathbb{D D}^{6}$ \\ ${ }^{1}$ Department of Mathematics, University of Sargodha, Sargodha, Pakistan \\ ${ }^{2}$ Department of Mathematics, Prince Sultan University, Riyadh, Saudi Arabia \\ ${ }^{3}$ Department of Medical Research, China Medical University, Taichung 40402, Taiwan \\ ${ }^{4}$ Department of Computer Science and Information Engineering, Asia University, Taichung 40402, Taiwan \\ ${ }^{5}$ Department of Mathematics and Statistics, Hazara University, Mansehra, Pakistan \\ ${ }^{6}$ Department of Mathematics, College of Arts and Sciences, Prince Sattam Bin Abdulaziz University, Wadi Aldawaser, \\ Saudi Arabia
}

Correspondence should be addressed to T. Abdeljawad; tabdeljawad@psu.edu.sa

Received 7 May 2020; Revised 30 July 2020; Accepted 17 August 2020; Published 19 December 2020

Academic Editor: Ljubisa Kocinac

Copyright ( $\odot 2020$ U. Ayub et al. This is an open access article distributed under the Creative Commons Attribution License, which permits unrestricted use, distribution, and reproduction in any medium, provided the original work is properly cited.

In this paper, we investigate some properties of the Pochhammer $(p, s, k)$-symbol ${ }_{p}[\xi]_{n, k, s}$ and gamma $(p, s, k)$-function ${ }_{p} \Gamma_{s, k}(\xi)$. We then prove several identities for newly defined symbol ${ }_{p}[\xi]_{n, k, s}$ and the function ${ }_{p} \Gamma_{s, k}(\xi)$. The integral representations for the gamma $(p, s, k)$-function and beta $(p, s, k)$-function are presented. Also, we define a new Mittag-Leffler $(p, s, k)$-function and study its analytic properties and its transforms.

\section{Introduction}

The theory of special functions comprises a major part of mathematics. In the last three centuries, the essential of solving the problems taking place in the fields of classical mechanics, hydrodynamics, and control theories motivated the development of the theory of special functions. This field also has wide applications in both pure mathematics and applied mathematics. The interested readers may consult the literature [1-4].

The Mittag-Leffler function takes place naturally similar to that of the exponential function in the solutions of fractional integro-differential equations having the arbitrary order. The Mittag-Leffler functions have to gain more recognition due to its wide applications in diverse fields. We suggest the readers to review the literature [5-16] for more details.

Throughout this article, let $\mathbb{C}, \mathbb{R}^{+}, \mathbb{Z}^{-}$, and $\mathbb{N}$ be the sets of complex numbers, positive real numbers, negative integers, and natural numbers, respectively.

\section{Preliminaries}

This section contains some basic definitions and mathematical preliminaries. We begin with the well-known Mittag-Leffler function.

In [17], Gosta Mittag-Leffler introduced the following Mittag-Leffler function which is defined by

$$
E_{\theta}(z)=\sum_{n=0}^{\infty} \frac{z^{n}}{\Gamma(\theta n+1)}, \quad z \in \mathbb{C}, \mathfrak{R}(\theta)>0 .
$$

In [18], a generalization of Mittag-Leffler function $E_{\theta}(z)$ (1) is given by

$$
E_{\theta, \vartheta}(z)=\sum_{n=0}^{\infty} \frac{z^{n}}{\Gamma(\theta n+\vartheta)}, \quad z, \vartheta \in \mathbb{C}, \mathfrak{R}(\theta)>0 .
$$

In [6], Prabhakar proposed the following three parameters of Mittag-Leffler function, which is defined by

$$
E_{\theta, \vartheta}^{\varrho}(z)=\sum_{n=0}^{\infty} \frac{z^{n}(\varrho)_{n}}{\Gamma(\theta n+\vartheta) n !}, \quad z, \vartheta, \varrho \in \mathbb{C}, \mathfrak{R}(\theta)>0,
$$


where $(\varrho)_{n}$ is the well-known Pochhammer's symbol defined as follows (see [19]). For $\varrho \in \mathbb{C}$,

$$
\begin{aligned}
(\varrho)_{n} & =\varrho(\varrho+1)(\varrho+2) \cdots(\varrho+n-1), \quad \text { for } n \in \mathbb{N} \\
& =1, \quad \text { for } n=0 .
\end{aligned}
$$

Definition 1. In [20], Gehlot introduced the two parameters Pochhammer's symbol and two parameters of gamma $(p, k)$-function.

Let $k, p>0, \xi \in \mathbb{C}$ with $\Re(\xi)>0$, and $n \in \mathbb{N}$. Then, the Pochhammer $(p, k)$-symbol ${ }_{p}(\xi)_{n, k}$ is defined as

$$
{ }_{p}(\xi)_{n, k}=\left(\frac{\xi p}{k}\right)\left(\frac{\xi p}{k}+p\right) \cdots\left(\frac{\xi p}{k}+(n-1) p\right)
$$

and the gamma $(p, k)$-function is defined by

$$
p^{\Gamma_{k}}(\xi)=\frac{1}{k} \lim _{n \longrightarrow \infty} \frac{n ! p^{n+1}(n p)^{\xi / k}}{p(\xi)_{n+1, k}} .
$$

Definition 2. The gamma $(p, k)$-function is also represented by the following forms:

$$
\begin{aligned}
& { }_{p} \Gamma_{k}(\xi)=\int_{0}^{\infty} e^{-t^{k} / p} t^{x-1} \mathrm{~d} t, \\
& { }_{p} \Gamma_{k}(\xi)=\left(\frac{p}{k}\right)^{\xi / k} \Gamma_{k}(\xi)=\frac{p^{\xi / k}}{k} \Gamma\left(\frac{\xi}{k}\right) .
\end{aligned}
$$

Also, the relationship between the Pochhammer $(p, k)$-symbol, Pochhammer $k$-symbol, and the classical Pochhammer's symbol is represented by [4]

$$
{ }_{p}(\xi)_{n, k}=\left(\frac{p}{k}\right)^{n}(\xi)_{n, k}=p^{n}\left(\frac{\xi}{k}\right)_{n} .
$$

The gamma $(p, k)$-function ${ }_{p} \Gamma_{k}(\xi)$ satisfies the following relation:

$$
p(\xi)_{n, k}=\frac{p \Gamma_{k}(\xi+n k)}{p \Gamma_{k}(\xi)}
$$

where

$$
{ }_{p} \Gamma_{k}(\xi+k)=\left(\frac{\xi p}{k}\right) \Gamma_{k}(\xi) .
$$

Definition 3. In [21], the Mittag-Leffler $k$-function is defined by

$$
E_{k, \theta, \vartheta}^{\varrho}(z)=\sum_{n=0}^{\infty} \frac{(\varrho)_{n, k} z^{n}}{\Gamma_{k}(\theta n+9) n !},
$$

where $k \in \mathbb{R}, z, \theta, \vartheta, \varrho \in \mathbb{C}, \mathfrak{R}(\theta)$, and $\Re(\vartheta)>0$.

Recently, Cerutti et al. [22] introduced the following Mittag-Leffler $(p, k)$-function is defined as follows.

Definition

$p, k \in \mathbb{R}, z, \theta, \vartheta, \varrho \in \mathbb{C}, \mathfrak{R}(\theta)>0$, and $\Re(\vartheta)>0$,

$$
{ }_{p} E_{k, \theta, \vartheta}^{\varrho}(z)=\sum_{n=0}^{\infty} \frac{p(\varrho)_{n, k} z^{n}}{p \Gamma_{k}(\theta n+\vartheta) n !}
$$

where ${ }_{p}(\varrho)_{n, k}$ is the Pochhammer $(p, k)$-symbol given by (5) and ${ }_{p} \Gamma_{k}(\xi)$ is gamma $(p, k)$-function given by (6).

In [23], Pochhammer $(p, s, k)$-symbol $p[\xi]_{n, k, s}$ is defined as follows.

Definition 5. For $p, k, \xi \in \mathbb{R}, 0<s<1$, and $n \in \mathbb{N}$,

$$
\begin{aligned}
{ }_{p}[\xi]_{n, k, s} & =\left[\frac{\xi p}{k}\right]_{s}\left[\frac{\xi p}{k}+p\right]_{s} \ldots\left[\frac{\xi p}{k}+(n-1) p\right]_{s} \\
& =\prod_{i=0}^{n-1}\left[\frac{\xi p}{k}+i p\right]_{s}
\end{aligned}
$$

where

$$
[\xi]_{s}=\frac{1-s^{\xi}}{1-s}, \quad \forall \xi \in \mathbb{R} .
$$

The following identities are satisfied:

(1) $[\xi+y]_{s}=[\xi]_{s}+s^{\xi}[y]_{s} \forall \xi, y \in \mathbb{R}$

(2) $[1]_{s}=1$

(3) $[\xi y]_{s}=[\xi]_{s^{y}}[y]_{s} \forall \xi, y \in \mathbb{R}$

(4) $[0]_{s}=0$

Definition 6. In [23], the gamma ( $p, s, k)$-function in term of $s$-series is given by

$$
\left.{ }_{p} \Gamma_{s, k}(k n)=\left[\frac{p}{k}\right]\right]_{s} \frac{\left(s^{p} ; s^{p}\right)_{n-1}}{(1-s)^{n-1}} .
$$
[23]

The relationship between ${ }_{p}[\xi]_{n, k, s}$ and ${ }_{p} \Gamma_{s, k}(\xi)$ is given by

$$
{ }_{p}[\xi]_{n, k, s}=\frac{p \Gamma_{s_{s k}}(\xi+n k)}{p \Gamma_{s, k}(\xi)} .
$$

The beta $(p, k)$-function is defined by

$$
{ }_{p} B_{k}(\xi, y)=\frac{p \Gamma_{k}(\xi)_{p} \Gamma_{k}(y)}{p \Gamma_{k}(\xi+y)}, \quad \mathfrak{R}(\xi)>0, \mathfrak{R}(y)>0 .
$$

Definition 7 . The beta $(p, k)$-function is represented by the following integral:

$$
{ }_{p} B_{k}(\xi, y)=\frac{1}{k} \int_{0}^{1} t^{(\xi / k)-1}(1-t)^{(y / k)-1} e^{-p / t} \mathrm{~d} t
$$

Definition 8. The well-known Laplace transform of piecewise continuous function $f: \mathbb{R} \longrightarrow \mathbb{R}$ is defined by

$$
\mathscr{L}\{f(\theta)\}=\int_{0}^{\infty} e^{-s \theta} f(\theta) \mathrm{d} \theta, \mathfrak{R}(\mathbf{s})>0 .
$$




\section{Applications and Properties of the Pochhammer $(p, s, k)$-Symbol ${ }_{p}[\xi]_{n, k, s}$ and Gamma $(p, s, k)$-Function ${ }_{p} \boldsymbol{\Gamma}_{s, k}(\xi)$}

In this section, we define gamma $(p, s, k)$-function ${ }_{p} \Gamma_{s, k}(\xi)$ in terms of limit function and give its integral representation. Also, we define beta $(p, s, k)$-function ${ }_{p} B_{s, k}(\xi, y)$ and its integral representation. Furthermore, we prove some identities of the Pochhammer $(p, s, k)$-symbol ${ }_{p}[\xi]_{n, k, s}$ and the gamma $(p, s, k)$-function ${ }_{p} \Gamma_{s, k}(\xi)$.

Definition 9. Suppose that $k, s, p>0, \xi \in \mathbb{C} / k \mathbb{Z}^{-}$with $\mathfrak{R}(\xi)>0$, and $n \in \mathbb{N}$. Then, the gamma $(p, s, k)$-function ${ }_{p} \Gamma_{s, k}(\xi)$ is given as

$$
{ }_{p} \Gamma_{s, k}(\xi)=\frac{s}{k} \lim _{n \longrightarrow \infty} \frac{n ! p^{n+1}(s n p)^{(\xi / k)-1}}{p(\xi)_{n, k}} .
$$

Theorem 1. Suppose that $k, p, s, r, q>0, \xi \in \mathbb{C} / k \mathbb{Z}^{-}$with $\mathfrak{R}(\xi)>0$, and $n \in \mathbb{N}$. Then, the following identities hold:

$$
\begin{aligned}
(1)_{{ }_{p}}[\xi]_{n, r, s} & ={ }_{p}\left[\frac{q \xi}{r}\right]_{n, q, s} \\
(2)_{{ }_{p}}[\xi]_{n, r, s} & =\left(\frac{p}{r}\right)^{n} r\left[\frac{q \xi}{r}\right]_{n, q, s} \\
\text { (3). }{ }_{p}[\xi]_{n, k, s} & ={ }_{s}[\xi]_{n, k, p} \\
(4)_{p_{p}} \Gamma_{s, k}(\xi) & =\left(\frac{q}{k}\right)_{p} \Gamma_{s, q}\left(\frac{q \xi}{k}\right) \\
\text { (5). }{ }_{r} \Gamma_{s, q}(\xi) & =\left(\frac{k}{q}\right)\left(\frac{r}{p}\right)^{\xi / s}{ }_{p} \Gamma_{s, k}\left(\frac{k \xi}{s}\right) \\
\text { (6). }{ }_{r} \Gamma_{k, q}(\xi) & =\left(\frac{r}{p}\right)^{\xi / q}{ }_{p} \Gamma_{k, q}(\xi)
\end{aligned}
$$

Proof. The properties (22), (23), and (24), respectively, follow from definition (11) and equations (2.8), (2.9), and (2.10) of [16]. The properties (25), (26), and (27), respectively, follow by using equation (21) and (2.11), (2.12), and (2.13) of [16].

Theorem 2. Let $k, p, s, r>0, \xi \in \mathbb{C} / k \mathbb{Z}^{-}$with $\Re(\xi)>0$, and $n \in \mathbb{N}$. Then, the following integral representation of gamma $(p, s, k)$-function is defined by

$$
{ }_{p} \Gamma_{s, k}(\xi)=\int_{0}^{\infty} e^{-t^{k} / s p} t^{\xi-1} \mathrm{~d} t .
$$

Proof. Consider the right hand side of (28). By applying ([8], page 2) Tannery theorem and using (21), we have $\int_{0}^{\infty} e^{-t^{k} / s p} t^{\xi-1} \mathrm{~d} t=\lim _{n \longrightarrow \infty} \int_{0}^{(s n p)^{1 / k}}\left(1-\frac{t^{k}}{n s p}\right)^{n} t^{\xi-1} \mathrm{~d} t$.

Let $C_{n, i}(\xi), i=0,1, \ldots, n$, be given by

$$
C_{n, i}(\xi)=\lim _{n \longrightarrow \infty} \int_{0}^{(s n p)^{1 / k}}\left(1-\frac{t^{k}}{n s p}\right)^{i} t^{\xi-1} \mathrm{~d} t .
$$

After integrating by parts, we obtain

$$
C_{n, i}(\xi)=\frac{k i}{n s p x} C_{n, i-1}(\xi+k), \quad \forall i=1,2, \ldots, n .
$$

Also,

$$
C_{n, 0}(\xi)=\frac{(s n p)^{\xi / k}}{\xi}
$$

Therefore,

$$
\begin{aligned}
C_{n, n}(\xi) & =\frac{s}{k} \frac{n ! p^{n+1}(s n p)^{(\xi / k)-1}}{(\xi)_{n, k}(1+(\xi / k n))}, \\
p^{\Gamma_{s, k}}(\xi) & =\lim _{n \longrightarrow \infty} C_{n, n}(\xi)=\frac{s}{k} \lim _{n \longrightarrow \infty} \frac{n ! p^{n+1}(s n p)^{(\xi / k)-1}}{p(\xi)_{n, k}} .
\end{aligned}
$$

Definition 10. Let $\xi, y \in \mathbb{C} / k \mathbb{Z}^{-}, k, p, s, r \in \mathbb{R}^{+}-0$, and $\mathfrak{R}(\xi)>0, n \in \mathbb{N}$. Then, the integral representation of ${ }_{p} B_{s, k}(\xi, y)$ is given by

$$
{ }_{p} B_{s, k}(\xi, y)=\frac{1}{k} \int_{0}^{1} t^{(\xi / k)-1}(1-t)^{(y / k)-1} e^{-(p / t)-(s /(1-t))} \mathrm{d} t,
$$

where $\mathfrak{R}(p)>0, \mathfrak{R}(s)>0, \mathfrak{R}(\xi)>0$, and $\mathfrak{R}(y)>0$.

Theorem 3. The relation between three parameters, two parameters, and the classical Pochhammer's symbol is given by

$$
{ }_{p}[\xi]_{n, k, s}=s_{p}^{n}(\xi)_{n, k}=\left(\frac{s p}{k}\right)^{n}(\xi)_{n, k}=(s p)^{n}\left(\frac{\xi}{k}\right)_{n} .
$$

Proof. Using (14) and (9), we get the desired result.

Theorem 4. The relation between gamma ( $p, s, k)$-function, gamma $(p, k)$-function, gamma $k$-function, and classic gamma function is given by

$$
{ }_{p} \Gamma_{s, k}(\xi)=(s)^{\xi / k}{ }_{p} \Gamma_{k}(\xi)=\left(\frac{s p}{k}\right)^{\xi / k} \Gamma_{k}(\xi)=\frac{(s p)^{\xi / k}}{k} \Gamma\left(\frac{\xi}{k}\right) .
$$

Proof. Using (21) and (8), we get the desired result. 
Theorem 5. Given $\xi \in \mathbb{C} k Z^{-}, k, s, p \in \mathbb{R}^{+}-\{0\}, \mathfrak{R}(\xi)>0$, and $n \in \mathbb{N}$, the recurrence relation for Pochhammer $(p, s, k)$-symbol is given by

$$
\begin{aligned}
{ }_{p}[\xi]_{n+j, k, s} & ={ }_{p}[\xi]_{j, k, s} \times_{p}[\xi+j k]_{n, k, s}, \\
(n s)_{p}[\xi]_{n-1, k, s} & ={ }_{p}[\xi]_{n, k, s}-{ }_{p}[\xi-k]_{n, k, s} .
\end{aligned}
$$

Proof. Using the definition of Pochhammer $(p, s, k)$-symbol, we get the desired result.

\section{Definition and Convergence Condition of the Mittag-Leffler $(p, s, k)$-Function ${ }_{p} E_{k, \theta, 9}^{\boldsymbol{Q}, s}(z)$}

In this section, we define a new generalization of the MittagLeffler $(p, s, k)$-function. Also, we check the convergence of the Mittag-Leffler $(p, s, k)$-function.

Definition 11. Suppose that $p, k \in \mathbb{R}, \theta, \vartheta, \varrho \in \mathbb{C}$, $\mathfrak{R}(\theta)>0, \mathfrak{R}(\vartheta)$, and $\mathfrak{R}(\varrho)>0$. Then, Mittag-Leffler $(p, s, k)$-function is defined by

$$
{ }_{p} E_{k, \theta, \vartheta}^{\varrho, s}(z)=\sum_{n=0}^{\infty} \frac{{ }_{p}[\varrho]_{n, k, s} z^{n}}{\Gamma_{s, k}(\theta n+\vartheta) n !},
$$

where ${ }_{p}[\varrho]_{n, k, s}$ is Pochhammer $(p, s, k)$-symbol defined in (14), and ${ }_{p} \Gamma_{s, k}(\xi)$ is defined in (21). The recurrence relation of gamma $(p, s, k)$-function ${ }_{p} \Gamma_{s, k}(\xi)$ given in [23] is

$$
{ }_{p} \Gamma_{s, k}(x+k)=\left[\frac{\xi p}{k}\right]_{s} \Gamma_{s, k}(\xi) .
$$

Now, some characteristics of the Mittag-Leffler $(p, s, k)$-function are presented. We show that the M-L $(p, s, k)$-function is an entire function. Also, its order and type are given.

Theorem 6. The Mittag-Leffler ( $p, s, k)$-function, defined in (38), is an entire function of order $\rho$ and type $\sigma$ given by

$$
\begin{aligned}
\rho & =\frac{k}{\operatorname{Re}(\theta)}, \\
\sigma & =\left[\rho p e^{\operatorname{Re}((\theta / k) \ln (\theta / k)) \rho}\right]^{-1} .
\end{aligned}
$$

Proof. Let $R$ denotes the radius of convergence of the Mittag-Leffler $(p, s, k)$-function. By considering the properties (5) and (8) and using the asymptotic expansions for the gamma function [1] and the asymptotic Stirling's formula, we have

$$
\begin{aligned}
\Gamma(z)= & (2 \pi)^{1 / 2} z^{z-(1 / 2)} e^{z}[1+O(z-1)] \\
& \cdot(|\arg (z)|<\pi ;|z| \longrightarrow \infty) .
\end{aligned}
$$

In particular,

$$
n !=(2 \pi n)^{1 / 2} n^{n} e^{-n}\left[1+O\left(n^{-1}\right)\right](n \in N ; n \longrightarrow \infty),
$$

and the following quotient expansion of two gamma functions at infinity is given as
$\frac{\Gamma(z+a)}{\Gamma(z+b)}=z^{a-b}\left[1+O\left(z^{-1}\right)\right](|\arg (z)+a|<\pi ;|z| \longrightarrow \infty)$.

Series (38) can be written in the following forms:

$$
\begin{aligned}
{ }_{p} E_{k, \theta, 9}^{\varrho, s}(z) & =\sum_{n=0}^{\infty} \frac{{ }_{p}[\varrho]_{n, k, s} z^{n}}{\Gamma_{s, k}(\theta n+\vartheta) n !} \\
& =\sum_{n=0}^{\infty} c_{n} z^{n},
\end{aligned}
$$

since

$$
R=\lim _{n \longrightarrow \infty} \sup \left|\frac{c_{n}}{c_{n+1}}\right| .
$$

In view of the properties (35) and (36) and using (III.9) of Theorem (1) in [22], we get

$$
\begin{aligned}
\left|\frac{c_{n}}{c_{n+1}}\right| & =\left|\frac{p[\varrho]_{n, k, s p} \Gamma_{s, k}(\theta(n+1)+\vartheta)(n+1) !}{{ }_{p}[\varrho]_{n+1, k, s} \Gamma_{s, k}(\theta n+\vartheta) n !}\right| \\
& \simeq\left|\frac{s^{n}{ }_{p}[\varrho]_{n, k} s^{(\theta n+\theta+9) / k}{ }_{p} \Gamma_{k}(\theta(n+1)+9)(n+1) !}{s^{n+1}{ }_{p}[\varrho]_{n+1, k} s_{p}^{(\theta n+9) / k} \Gamma_{k}(\theta n+\vartheta) n !}\right| \\
& \simeq(n+1) s^{9 / k} p^{(\theta / k)-1}\left(\frac{\theta n}{k}\right)^{\theta / k} \longrightarrow \infty .
\end{aligned}
$$

Thus, the Mittag-Leffler $(p, s, k)$-function is an entire function.

To obtain the order $\rho$ and the type $\sigma$, we apply the following definitions of $\rho$ and $\sigma$, respectively:

$$
\begin{gathered}
\rho=\lim _{n \longrightarrow \infty} \sup \frac{n \ln n}{\ln \left(1 /\left|c_{n}\right|\right)}, \\
e^{\rho} \sigma=\lim _{n \longrightarrow \infty} \sup \left(n\left|c_{n}\right|^{\rho / n}\right) .
\end{gathered}
$$

Consider

$$
\begin{aligned}
\frac{1}{\left|c_{n}\right|} & =\left|\frac{p \Gamma_{s, k}(\varrho)_{p} \Gamma_{s, k}(\theta n+\vartheta) n !}{{ }_{p} \Gamma_{s, k}(\varrho+n k)}\right| \\
& =s^{(\theta(n-1)+9) / k}\left|\frac{\Gamma_{k}(\varrho)_{p} \Gamma_{k}(\theta n+\vartheta) n !}{p \Gamma_{k}(\varrho+n k)}\right| .
\end{aligned}
$$

By using Theorem (1) equation (III.12) ([22]) and definition of $\rho$ (47), we get

$$
\rho=\frac{k}{\operatorname{Re}(\theta)} .
$$

Similarly, by putting the value of $\left|c_{n}\right|$ in the definition of $\sigma(48)$ and simplify as the same in Theorem (1) in equation (III.14) ([22]), we obtain

$$
\sigma=\left[\rho p e^{\Re((\theta / k) \ln (\theta / k)) \rho}\right]^{-1} .
$$




\section{Applications and Properties of the Mittag- Leffler $(p, s, k)$-Function ${ }_{p} E_{k, \boldsymbol{\theta}, \boldsymbol{9}}^{\boldsymbol{Q}, s}(z)$}

Some basic properties of Mittag-Leffler $(p, s, k)$-function ${ }_{p} E_{k, \theta, 9}^{0, s}(z)$ are presented in this section.

Theorem 7. Suppose that $p, k, s \in \mathbb{N}, \theta, \vartheta, \varrho \in \mathbb{C}$ with $\mathfrak{R}(\theta)>0, \mathfrak{R}(\vartheta)$ and $\mathfrak{R}(\varrho)>0$, then

(1)

$$
\left(\frac{\mathrm{d}}{\mathrm{d} z}\right)^{m}{ }_{p} E_{k, \theta, \vartheta}^{\mathrm{Q}, s}(z)={ }_{p}[\varrho]_{m, k, s p} E_{k, \theta, \theta m+\vartheta}^{\varrho+m k, s}(z) .
$$

(2)

$$
\begin{aligned}
\left(\frac{\mathrm{d}}{\mathrm{d} z}\right)^{m}\left(z^{9 / k}\right)_{p} E_{k, \theta, \vartheta}^{\varrho, s}\left(z^{\theta / k}\right)= & z^{((\theta n / k)-m+(\vartheta / k)-1)} \\
& \cdot{ }_{p}[\varrho]_{m, k, s p} E_{k, \theta, \theta m+\vartheta}^{\varrho+m k, s}\left(z^{\theta / k}\right) .
\end{aligned}
$$

Proof. (1) Taking L.H.S of (42),

$$
\begin{aligned}
\left(\frac{\mathrm{d}}{\mathrm{d} z}\right)^{m}{ }_{p} E_{k, \theta, \vartheta}^{\varrho, s}(z) & =\left(\frac{\mathrm{d}}{\mathrm{d} z}\right)^{m}\left(\sum_{n=0}^{\infty} \frac{{ }_{p}[\varrho]_{n, k, s} z^{n}}{\Gamma_{s, k}(\theta n+\vartheta) n !}\right) \\
& =\sum_{n=m}^{\infty} \frac{{ }_{p}[\varrho]_{n, k, s} z^{n-m}}{\Gamma_{s, k}(\theta n+\vartheta)(n-m) !} \\
& =\sum_{n=0}^{\infty} \frac{p}{{ }_{p}[\varrho]_{n+m, k, s}(\theta(n+m)+\vartheta) n !} .
\end{aligned}
$$

By using

$$
{ }_{p}[\xi]_{n+j, k, s}={ }_{p}[\xi]_{j, k, s} \times{ }_{p}[\xi+j k]_{n, k, s},
$$

in the above equation, we get the desired result (52).

(2) Taking L.H.S of (43),

$$
\begin{aligned}
\left(\frac{\mathrm{d}}{\mathrm{d} z}\right)^{m}\left(z_{p}^{9 / k} E_{k, \theta, \vartheta}^{\varrho, s}\left(z^{\theta / k}\right)\right) & =\left(\frac{\mathrm{d}}{\mathrm{d} z}\right)^{m}\left(\sum_{n=0}^{\infty} \frac{{ }_{p}[\varrho]_{n, k, s} z^{(\theta n / k)+(\vartheta / k)-1}}{p^{\Gamma_{s, k}}(\theta n+\vartheta) n !}\right) \\
& =\sum_{n=m}^{\infty} \frac{p[\varrho]_{n, k, s} z^{(\theta n / k)+(\vartheta / k)-m-1}}{p^{\Gamma_{s, k}}(\theta n+\vartheta)(n-m) !} \\
& =\sum_{n=0}^{\infty} \frac{{ }_{p}[\varrho]_{n+m, k, s} z^{\theta / k)(n+m+(\vartheta / k)-m-1}}{p_{q, k}(\theta(n-m)+\vartheta) n !} .
\end{aligned}
$$

By using

$$
{ }_{p}[\xi]_{n+j, k, s}={ }_{p}[\xi]_{j, k, s} \times{ }_{p}[\xi+j k]_{n, k, s},
$$

in the above equation, we get the desired result (53).

Theorem 8. Suppose that $k>0$ and $\theta, \vartheta$ and $\varrho \in \mathbb{C}$ with $\mathfrak{R}(\theta)>0, \mathfrak{R}(\vartheta)>0$ and $\mathfrak{R}(\varrho)>0$, then

$$
\int_{0}^{z} t^{(9 / k)-1}{ }_{p} E_{k, \theta, \vartheta}^{0, s}\left(a t^{\theta / k}\right) \mathrm{d} t=s^{(\theta n+\vartheta) / k} p z^{9 / k}{ }_{p} E_{k, \theta,(9+k)}^{0, s}\left(a z^{\theta / k}\right) .
$$

Proof. Consider the L.H.S.

$$
\int_{0}^{z} t^{(\vartheta / k)-1}{ }_{p} E_{k, \theta, \vartheta}^{\varrho, s}\left(a t^{\theta / k}\right) \mathrm{d} t=\int_{0}^{z} t^{(\vartheta / k)-1} \sum_{n=0}^{\infty} \frac{p_{p}[\varrho]_{n, k, s}\left(a t^{\theta / k}\right)^{n}}{\Gamma_{s, k}(\theta n+\vartheta) n !} .
$$

By changing integration and summation orders in the above equation, we have

$$
\begin{aligned}
\int_{0}^{z} t_{p}^{(9 / k)-1} E_{k, \theta, \vartheta}^{\varrho, s}\left(a t^{\theta / k}\right) \mathrm{d} t= & \sum_{n=0}^{\infty} \frac{{ }_{p}[\varrho]_{n, k, s}(a)^{n}}{\Gamma_{s, k}(\theta n+\vartheta) n !} \\
& \cdot \int_{0}^{z} t^{(\theta n / k)+(\vartheta / k)-1} \mathrm{~d} t \\
= & \sum_{n=0}^{\infty} \frac{{ }_{p}[\varrho]_{n, k, s}(a)^{n} z^{(\theta n / k)+(\vartheta / k)}}{((\theta n+\vartheta) / k)_{p} \Gamma_{s, k}(\theta n+\vartheta) n !} .
\end{aligned}
$$

By using the recurrence relation of ${ }_{p} \Gamma_{s, k}(\xi)$ and ${ }_{p} \Gamma_{k}(\xi)$ and equation (III.31) in [22], we get

$$
\left(\frac{\theta n+\vartheta}{k}\right)_{p} \Gamma_{s, k}(\theta n+\vartheta)=\frac{s^{(\theta n+\vartheta) / k}}{p}{ }_{p} \Gamma_{s, k}(\theta n+\vartheta+k) .
$$

By using the above result in equation (60), we have

$$
\int_{0}^{z} t_{p}^{(\vartheta / k)-1} E_{k, \theta, \vartheta}^{\varrho, s}\left(a t^{\theta / k}\right) \mathrm{d} t=s^{(\theta n+9) / k} p z_{p}^{\vartheta / k} E_{k, \theta, 9+k}^{0, s}\left(a z^{\theta / k}\right) \text {. }
$$

\section{The Euler-Beta Transform of the Mittag- Leffler $(p, s, k)$-Function ${ }_{p} E_{k, \theta, 9}^{o, s}(z)$}

The well-known Euler-beta transform is defined by

$$
\mathbf{B}\{f(\theta) ; a ; b\}=\int_{0}^{1} \theta^{a-1}(1-\theta)^{b-1} f(\theta) \mathrm{d} \theta,
$$

where $a, b \in \mathbb{R}$ and $\min \{\mathfrak{R}(\theta), \mathfrak{R}(\vartheta)\}>0$.

Next, we define the beta transform of newly defined M-L function.

Theorem 9. Suppose that $p, s, k>0$ and $\theta, \vartheta, \varrho, a, b \in \mathbb{C}$ with $\mathfrak{R}(\theta)>0, \mathfrak{R}(\vartheta)>0, \mathfrak{R}(\varrho)>0, \mathfrak{R}(a)>0$, and $\mathfrak{R}(b)>0$, then 


$$
\mathbf{B}\left({ }_{p} E_{k, \theta, \vartheta}^{\mathrm{Q}, s}\left(\lambda z^{\theta / k}\right) ; \frac{\vartheta}{k} ; \frac{b}{k}\right)=k_{p} \Gamma_{s, k}(b)_{p} E_{k, \theta, \vartheta+b}^{0, s}(\lambda) .
$$

Proof.

$$
\begin{aligned}
\mathbf{B}\left({ }_{p} E_{k, \theta, \vartheta}^{\varrho, s}\left(\lambda z^{\theta / k}\right) ; \frac{\vartheta}{k} ; \frac{b}{k}\right)= & \int_{0}^{1} z^{(\vartheta / k)-1}(1-z)^{(b / k)-1} \\
& \cdot \sum_{n=0}^{\infty} \frac{p}{{ }_{p}[\varrho]_{n, k, s} \lambda_{s, k}(\theta n+\vartheta) n !} \mathrm{d} z .
\end{aligned}
$$

After interchanging the integration and summation orders of the above equation and using the relation between the gamma $(p, s, k)$-function and the classical gamma function given by (3.4), we have

$$
\begin{aligned}
\mathbf{B}\left({ }_{p} E_{k, \theta, \vartheta}^{\varrho, s}\left(\lambda z^{\theta / k}\right) ; \frac{\vartheta}{k} ; \frac{b}{k}\right) & =\sum_{n=0}^{\infty} \frac{{ }_{p}[\varrho]_{n, k, s} \lambda^{n}}{\Gamma_{s, k}(\theta n+\vartheta) n !} \int_{0}^{1} z^{(\theta n / k)+(\vartheta / k)-1}(1-z)^{(b / k)-1} \mathrm{~d} z \\
& =\sum_{n=0}^{\infty} \frac{{ }_{p}[\varrho]_{n, k, s} \lambda^{n}}{\Gamma_{s, k}(\theta n+\vartheta) n !} \frac{\Gamma((\theta n+\vartheta) / k) \Gamma(b / k)}{\Gamma((\theta n+\vartheta+b) / k)} \\
& =\sum_{n=0}^{\infty} \frac{{ }_{p}[\varrho]_{n, k, s} \lambda^{n}}{\Gamma((\theta n+\vartheta) / k) n !} \frac{\Gamma((\theta n+\vartheta) / k) \Gamma(b / k)}{\Gamma((\theta n+\vartheta+b) / k)} .
\end{aligned}
$$

After simplification, we obtain the desired result:

$$
\mathbf{B}\left({ }_{p} E_{k, \theta, \vartheta}^{\varrho, s}\left(z^{\theta / k}\right) ; \frac{\vartheta}{k} ; \frac{b}{k}\right)=k_{p} \Gamma_{s, k}(b)_{p} E_{k, \theta, \vartheta+b}^{\varrho, s}(\lambda) .
$$

$$
\mathscr{L}\left\{z_{p}^{(\vartheta / k)-1} E_{k, \theta, \vartheta}^{\varrho, s}\left( \pm(c z)^{\theta / k}\right)\right\}=k(s p \mathbf{s})^{-\vartheta / k}\left[1 \mp p\left(\frac{c}{s k \mathbf{s}}\right)^{\theta / k}\right]^{-\varrho / k} .
$$

Remark 1 .

(i) If $s=1$, then equation (64) coincides with the result of [22], sec (IV.2)

(ii) If $s=1$ and $p=k$, then equation (64) coincides with the formula $(11,37)$ in $[21]$

(iii) If $s=p=k=1$, then equation (64) coincides with the formula (2.2.14) in [24]

\section{The Laplace Transform of the Mittag-Leffler $(p, s, k)$-Function ${ }_{p} E_{k, \boldsymbol{\theta}, \boldsymbol{\theta}}^{\boldsymbol{Q}, \boldsymbol{s}}(z)$}

Theorem 10. Let $p, s, k, c>0$ and $\theta, \vartheta, \varrho \in \mathbb{C}$ with $\mathfrak{R}(\theta)>0, \mathfrak{R}(\vartheta)>0$, and $\mathfrak{R}(\varrho)>0$. Then, the Laplace transform of $E_{p, \theta, \vartheta}^{0, s}$ is given by
Proof. Applying the Laplace transform on the left hand side of (68), the Laplace transform of the potential function (see [1], equation (1.4.58)) and the generalized binomial formula is given by

$$
(1-k w)^{-\varrho / k}=\sum_{k=0}^{\infty} \frac{(\varrho)_{n, k} w^{n}}{n !}
$$

We have

$$
\begin{aligned}
\mathscr{L}\left\{z^{(9 / k)-1}{ }_{p} E_{k, \theta, \vartheta}^{\varrho, s}\left( \pm(c z)^{\theta / k}\right)\right\} & =\int_{0}^{\infty} e^{-s z} z^{(9 / k)-1} \sum_{n=0}^{\infty} \frac{{ }_{p}[\varrho]_{n, k, s}}{\Gamma_{s, k}(\theta n+\vartheta) n !}( \pm 1)^{n}\left((c z)^{\theta n / k}\right) \mathrm{d} z \\
& =\sum_{n=0}^{\infty} \frac{p[\varrho]_{n, k, s}( \pm 1)^{n}(c)(\theta n / k)}{{ }_{p} \Gamma_{s, k}(\theta n+\vartheta) n !} \int_{0}^{\infty} e^{-s z} z^{(\theta n / k)+(\vartheta / k)-1} \mathrm{~d} z \\
& =\frac{k}{(s p \mathbf{s})^{9 / k}} \sum_{n=0}^{\infty} \frac{{ }_{p}[\varrho]_{n, k, s}}{n !(c / s p \mathbf{s})^{\theta n / k}} .
\end{aligned}
$$



have

Now, using the relation between ${ }_{p}[\varrho]_{n, k, s}$ and ${ }_{p}(\varrho)_{n, k}$, we

$$
\begin{aligned}
\mathscr{L}\left\{z^{(9 / k)-1} E_{k, \theta, \vartheta}^{\varrho, s}\left( \pm(c z)^{\theta / k}\right)\right\} & =\frac{k}{(s p \mathbf{s})^{9 / k}} \sum_{n=0}^{\infty} \frac{s_{p}^{n}[\varrho]_{n, k}}{n !}\left(\frac{c}{s p \mathbf{s}}\right)^{\theta n / k} \\
& =\frac{k}{(s p \mathbf{s})^{9 / k}} \sum_{n=0}^{\infty} \frac{s^{n}(p / k)^{n}(\varrho)_{n, k}}{n !}\left(\frac{c}{s p \mathbf{s}}\right)^{\theta n / k} \\
& =\frac{k}{(s p \mathbf{s})^{9 / k}} \sum_{n=0}^{\infty} \frac{(\varrho)_{n, k}}{n !}\left[\left(\frac{c}{s p \mathbf{s}}\right)^{\theta / k} \frac{s p}{k}\right]^{n} .
\end{aligned}
$$

Writing the series in compact form, we have

$$
\mathscr{L}\left\{z^{(\vartheta / k)-1}{ }_{p}^{\rho_{k, \theta, \vartheta}, s}\left( \pm(c z)^{\theta / k}\right)\right\}=\frac{k}{(s p \mathbf{s})^{9 / k}}\left(1 \mp p \mathbf{s}\left(\frac{c}{k s}\right)^{\theta / k}\right)^{-\varrho / k} \text {. }
$$

Remark 2. (a) If $s=1$ in the above theorem, then the result coincides with the formula of [22]

(b) If $p=s=k=c=1$ in Theorem 10 , then

$$
\mathscr{L}\left\{z^{\vartheta-1}{ }_{1} E_{1, \theta, \vartheta}^{\varrho, 1}\left(z^{\theta}\right)\right\}=\mathbf{s}^{\vartheta}\left(1-\mathbf{s}^{-\theta}\right)^{-\varrho},
$$

which coincides with formula (11.13) of [25].

\section{Conclusion}

In this paper, we established a new extension of the MittagLeffler function and investigated some of its properties. We concluded as follows:

(1) If $s=1$, then we get the results of the Mittag-Leffler $(p, s, k)$-function defined in [22]

(2) If $p=1$ and $s=1$, then we get the results of the Mittag-Leffler $k$-function defined in [21]

(3) If $k=1, p=1$, and $s=1$, then we get the results of the Mittag-Leffler defined in [6]

(4) If $p=1, k=1, s=1$, and $\varrho=1$, then we get the results of the Mittag-Leffler defined in [18]

(5) If $p=1, k=1, s=1, \vartheta=1$, and $\varrho=1$, then we get the results of the Mittag-Leffler $k$-function defined in [17]

(6) If $p=1, k=1, s=1, \theta=1, \vartheta=1$, and $\varrho=1$, then we get the exponential function

Recently, the Mittag-Leffler function is used to construct the fractional operators with nonsingular kernels [26, 27]. In $[28,29]$, the authors introduced the generalized fractional integrals and differential operators, which contain the Mittag-Leffler $k$-function in the kernels, and proved their various properties. Recently, Samraiz et al. [30] introduced the Hilfer Prabhakar $(k ; s)$-fractional derivative by using the Mittag-Leffler $k$-function. They discussed its various properties and the generalized Laplace transform of the said operator. They also discussed the applications of Hilfer Prabhakar's $(k ; s)$-derivative in mathematical physics. In this study, we defined further generalization of the MittagLeffler and proved its various basic properties. Hence, it would be of great interest that the Mittag-Leffler function studied in this article will be utilized to generalize such classes of fractional and differential operators.

\section{Data Availability}

No data were used to support the findings of the study.

\section{Conflicts of Interest}

The authors declare that they have no conflicts of interest.

\section{Authors' Contributions}

U.M. gave the main idea, and all other authors contributed equally to improve the final manuscript.

\section{Acknowledgments}

The author T. Abdeljawad would like to thank Prince Sultan University for funding this work through research group Nonlinear Analysis Methods in Applied Mathematics (NAMAM) (group number RG-DES-2017-01-17).

\section{References}

[1] A. A. Kilbas, H. M. Srivastava, and J. Trujillo, Theory and Application of Fractional Differential Equations, North-Holland Mathematics Studies, Vol. 207, Elsevier, Amsterdam, Netherlands, 2006.

[2] I. Podlubny, Fractional Differential Equations, Academic, New York, NY, USA, 1999.

[3] G. Gasper and M. Rahman, Basic Hypergeometric Series, Cambridge University Press, Cambridge, UK, 1990.

[4] A. Mathai and H. Haubold, Special Functions for Applied Scientists, Springer-Verlag, New York, NY, USA, 2008. 
[5] R. Gorenflo, A. A. Kilbas, and S. Rogosin, "On the generalized Mittag-Leffler type functions," Integral Transforms and Special Functions, vol. 7, no. 3-4, pp. 215-224, 1998.

[6] T. A. Prabhakar, "Singular integral equation with a generalized Mittag-Leffler function in the kernel," Yokohama Mathematical Journal, vol. 19, pp. 7-15, 1971.

[7] K. Gehlot, "The generalized k-Mittag-Leffler function," International Journal of Contemporary Mathematical Sciences, vol. 7, no. 45, pp. 2213-2219, 2012.

[8] A. Erdelyi, Higher Transcendental Function, McGraw-Hill Book Company, New York, NY, USA, 1953.

[9] R. Garra, R. Gorenflo, F. Polito, and Ž. Tomovski, "Hilferprabhakar derivatives and some applications," Applied Mathematics and Computation, vol. 242, pp. 576-589, 2014.

[10] H. M. Srivastava and Z. Tomovski, "Fractional calculus with an integral operator containing a generalized mittag-leffler function in the kernel," Applied Mathematics and Computation, vol. 211, no. 1, pp. 198-210, 2009.

[11] M. Garg, A. Sharma, and P. Manohar, "A generalized mittagleffler type function with four parameters," Thai Journal of Mathematics, vol. 14, no. 3, pp. 637-649, 2016.

[12] R. Gorenflo, A. A. Kilbas, F. Mainardi, and S. V. Rogosin, "Mittag-Leffler functions, related topics and applications," Monographs in Mathematics, Springer, Berlin, Germany, 2014.

[13] F. Mainardi, "On the advent of fractional calculus in econophysics via continuous-time random walk," Mathematics, vol. 8, no. 4, p. 641, 2020.

[14] E. Scalas, "Five years of continuous-time random walks ineconophysics," in, A. Namatame, T. Kaizouji, and Y. Aruka, Eds., Lecture Notes in Economics and Mathematical System, vol. 567, Springer, Berlin, Heidelberg, 2006.

[15] T. Skovranek, "The mittag-leffler fitting of the phillips curve," Mathematics, vol. 7, no. 7, p. 589, 2019.

[16] Y. Li, Y. Chen, and I. Podlubny, "Mittag-Leffler stability of fractional order nonlinear dynamic systems," Automatica, vol. 45, no. 8, pp. 1965-1969, 2009.

[17] G. M. Mittag-Leffler, "Sur la nouvelle fonction $E_{\theta_{1}}(\zeta)$," Comptes Rendus de l'Académie des Sciences, vol. 137, pp. 554-558, 1903.

[18] A. Wiman, "Über den fundamentalsatz in der teorie der funktionen $E_{a}(x)$," Acta Mathematica, vol. 29, pp. 191-201, 1905.

[19] H. Srivastava and J. Choi, Zeta and q-Zeta Functions and Associated Series and Integrals, Elsevier Science Publishers, London, UK, 2012.

[20] K. Gehlot, "Two parameter gamma function and its properties," 2017.

[21] G. Dorrego and R. Cerutti, "The k-Mittag-Leffler function," International Journal of Contemporary Mathematical Sciences, vol. 7, pp. 705-716, 2012.

[22] R. A. Cerutti, L. L. Luque, and G. A. Dorrego, "On the $(p-k)$-Mittag-Leffler function," Applied Mathematical Sciences, vol. 11, pp. 2541-2560, 2017.

[23] K. Gehlot and K. Nantomah, " $(p-q-k)$ gamma and beta functions and their properties," International Journal of Pure and Applied Mathematics, vol. 118, no. 3, pp. 519-526, 2018.

[24] A. Mathai, R. Saxena, and H. Houbold, The H-Function: Theory and Applications, Springer, Berlin, Heidelberg, 2010.

[25] H. J. Haubold and A. M. Mathai, "The fractional kinetic equation and thermonuclear functions," Astrophysics and Space Science, vol. 273, no. 1-4, pp. 53-63, 2000.
[26] A. Atangana and D. Baleanu, "New fractional derivative with non-local and non-singular kernel," Thermal Science, vol. 20, no. 2, pp. 757-763, 2016.

[27] T. Abdeljawad, "Fractional operators with generalized MittagLeffler kernels and their iterated differintegrals," Chaos: An Interdisciplinary Journal of Nonlinear Science, vol. 29, no. 2, Article ID 023102, 2019.

[28] G. A. Dorrego, "Generalized riemann-liouville fractional operators associated with a generalization of the prabhakar integral operator," Progress in Fractional Differentiation and Applications, vol. 2, no. 2, pp. 131-140, 2016.

[29] K. Nisar, G. Rahman, D. Baleanu, S. Mubeen, and M. Arshad, "The $(k, s)$-fractional calculus of $k$-Mittag-Leffler function," Advances in Difference Equations, vol. 118, no. 2017, 2017.

[30] M. Samraiz, Z. Parveen, G. Rahman, K. S. Nisar, and D. Kumar, "On $(k, s)$-Hilfer prabhakar fractional derivative with applications in mathematical physics," Frontiers in Physics, vol. 8, 2020. 